\title{
Thermal measurements of integrated inductors in CMOS technology and simple analytical model of heat conduction
}

\author{
by I. Papagiannopoulos*, ${ }^{\star \star}$, M. Kaluza**, G. De Mey***, V. Chatziathanasiou*, B. Wiecek**, and A. \\ Hatzopoulos*, \\ * Aristotle University of Thessaloniki, Department of Electrical and Computer Engineering, 54124 Thessaloniki, \\ Greece, ipapagia@auth.gr \\ ** Technical University of Lodz, Institute of Electronics, ul Wolczanska 211-215, 90-924 Lodz, Poland \\ Ghent, Belgium \\ *** Gent University, Department of Electronics and Information Systems, Sint Pietersnieuwstraat 41, 9000
}

\begin{abstract}
In this study, infrared thermography is used to measure the temperature response of integrated on silicon spiral inductors to a unit step electrical signal used to excite the samples. The measurements are performed with the use of high speed infrared equipment. The thermal impedance is then extracted from the transient temperature response. Finally, an attempt is made to model the thermal network of the spiral inductors based on the experimental measurements. A one-dimensional analytic thermal modelling approach is applied. Simple geometric structures are modelled in order to understand the complexity of the heat flow throughout the volume of the device under test.
\end{abstract}

\section{Introduction}

In the present work, the investigation of the transient thermal behaviour of spiral inductors integrated on silicon is presented. The nature of the problem is twofold. The first goal of the research consists of measuring the transient thermal response of the samples under test to a unit step electrical signal. Besides, the behaviour captured from the experiments is attempted to be modelled by means of simple one-dimensional heat diffusion equations.

A series of inductors has been designed and integrated in silicon AMIS 0.5, TM1P twin-tub technology [1] with three layers of metallization. A layout of the circuitry is presented in figure 1(a), and (c). The same set of inductors has been examined in the past in [2]. In the current study, the position of the inductors is the differentiating parameter between the different coils. For each inductor, their temperature response to a heat step function is measured. The inductors are excited by a DC current. The joule losses act as a power step function which excites the thermal RC network.

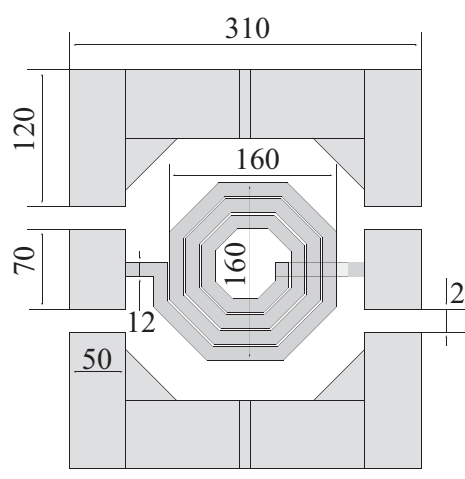

(a)

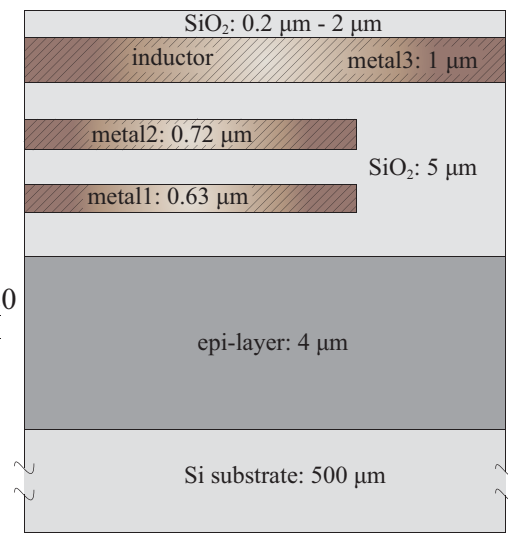

(b)

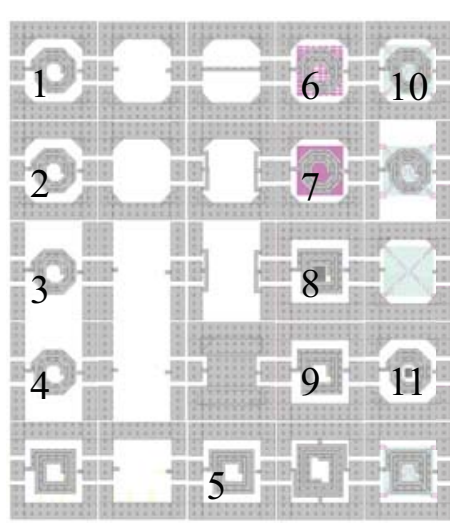

(c)

Fig. 1. (a) Layout of a sample inductor, (b) Cross sectional view of the wafer, (c) Layout of the integrated circuit

The thermal impedance of the device is consequently extracted from transient temperature measurements. These measurements were conducted with the use of high speed infrared equipment. The time domain measurements were transformed in the frequency domain. Nyquist representations of the complex thermal impedance were then plotted in order to evaluate the behaviour of the thermal RC network of the samples [3]. In this way, it is easier to observe the topography of the thermal network.

Having evaluated the thermal behaviour of the samples, an attempt has been made to model the heat flow. Simple analytical models of the heat transfer throughout the device are examined. The proposed modelling method is considering the structure as one-dimensional. With the aim of providing quick estimations of the thermal resistance or the dynamic thermal behaviour of electronic devices, various approximations have been used in previous works [4]. In the 
work of Vermeersch [5, 6], a one-dimensional approach was used to describe the heat diffusion in real cooled substrate with a square heat source on the top surface.

There also exist many exact or approximate solutions of the thermal problem of a heat source on a conductive substrate [7, 8]. The effect of heat sources' positioning on the thermal behaviour of the power dissipating elements is considered in [9]. Most of the above cited models are limited to the steady state heat conduction, suitable therefore to evaluate the thermal resistance. Most sophisticated approaches, addressed to the dynamic thermal behaviour modelling of electronic devices are also proposed in [10]. Similar techniques have also been used to create compact thermal models out of transient temperature measurements [11].

\section{Problem statement}

As shown in figure 1(a), the external dimensions of the geometry are approximately $160 \mu \mathrm{m} \times 160 \mu \mathrm{m}$. The silicon wafer is mounted on a FR4 board. The inductors are placed on top of a non-conductive $\mathrm{SiO}_{2}$ layer which constitutes a necessary electrical insulation between the inductor and the electrically conducting substrate. At the same time, the $\mathrm{SiO}_{2}$ layer is characterized by a much lower thermal conductivity in comparison to Si. Thus, the oxide layer constitutes also an unwanted thermal insulation layer [12]. Wire bonding is connecting the pads of each inductor with the FR4 copper coating, providing conductive path to the power supply. The inductors that were measured are 1, 2, and 3 in figure 1(c). The material properties which were used during the analytical calculations are given in Table 1.

Table 1. Properties of the different layers of the sample.

\begin{tabular}{cccccc}
\hline \hline \multicolumn{2}{c}{ Layer } & 1 & 2 & 3 & 4 \\
\hline \multicolumn{2}{c}{ Material } & paint & $\mathrm{SiO}_{2}$ & $\mathrm{Si}$ & $\mathrm{FR} 4$ \\
$c_{V}$ & {$\left[\mathrm{~J} / \mathrm{m}^{3} . \mathrm{K}\right]$} & 2000000 & 1613333 & 1654300 & 1110000 \\
$K$ & {$[\mathrm{~W} / \mathrm{m} . \mathrm{K}]$} & 1 & 1.38 & 148 & 0.25 \\
thickness & {$[\mu \mathrm{m}]$} & 5 & 5 & 350 & 1600 \\
\hline
\end{tabular}

It is common knowledge that there is a direct analogy between electrical and thermal networks. Trying to measure the thermal impedance though, one can notice that this is not an easy task. In the case of thermal engineering, measurement of the transient temperature response to a power step excitation is required.

Thermal impedance $Z_{t h}$ is a convenient parameter for the thermal characterization of electronic integrated circuits $[3,13]$. The temperature response $T_{\text {step }}(t)$ to a heating step function $P_{\text {ste }}(t)=P_{0} u(t)$ has been used to evaluate the thermal impedance versus frequency. The complex thermal impedance can be expressed by the following equation:

$$
Z_{\text {th }}(s)=\frac{T_{\text {step }}(s)}{P_{\text {step }}(s)}=\frac{L\left\{T_{\text {step }}(t)\right\}}{L\left\{P_{\text {step }}(t)\right\}}=\frac{s}{P_{0}} \int_{0}^{\infty} T_{\text {step }}(t) e^{-s t} d t \text {, }
$$

where $L$ denotes Laplace transformation, and $P_{0}$ is the power delivered to the inductor in steady state. By substituting $s=j \omega$ we get the frequency domain representation, where $\omega$ is the angular frequency.

In order to evaluate the thermal impedance, the temperature is measured on the heat source. In case of experimental measurements, a finite number of discrete temperature samples are available. Thus, the thermal impedance of equation (1) has to be calculated numerically. The algorithm which is proposed for the numerical calculation of the thermal impedance from the discrete measurements makes use of the Filon-type quadrature [14].

For the theoretical analysis, the samples were assumed to be one-dimensional (figure 2(a)). It was also proposed that the shape of the coils can be considered circular with homogeneous heat dissipation. It should be outlined that the position of the heat source on top of the chip die was not considered of importance during the analysis and therefore neglected from our analysis. Otherwise, it would be impossible to model the position of the heat source with the use of one-dimensional heat equation. The heat transfer coefficient $h$ is considered equal to $20 \mathrm{~W} / \mathrm{m}^{2} \mathrm{~K}$. The heat is supposed to spreading from a circular homogeneous heat source which is placed at the paint $/ \mathrm{SiO}_{2}$ interface. The radius of this circle is considered initially equal to $80 \mu \mathrm{m}$. The power $P$ dissipated is calculated as the product of the square of the current flowing through the series resistance of the inductor $I^{2}$ multiplied by the series resistance $R_{\text {series. }}$. The current is chosen equal to the one measured during the one measured during the experimental inspection of the samples.

\section{Experimental measurements}

The temperature response to a heat step function and thus the thermal impedance of the different heat sources can be used to describe the thermal network as well as the differences among them. For each inductor, their temperature response was measured. A DC current source was used as a heat source, and the value of the current was selected $99.6 \mathrm{~mA}$. 


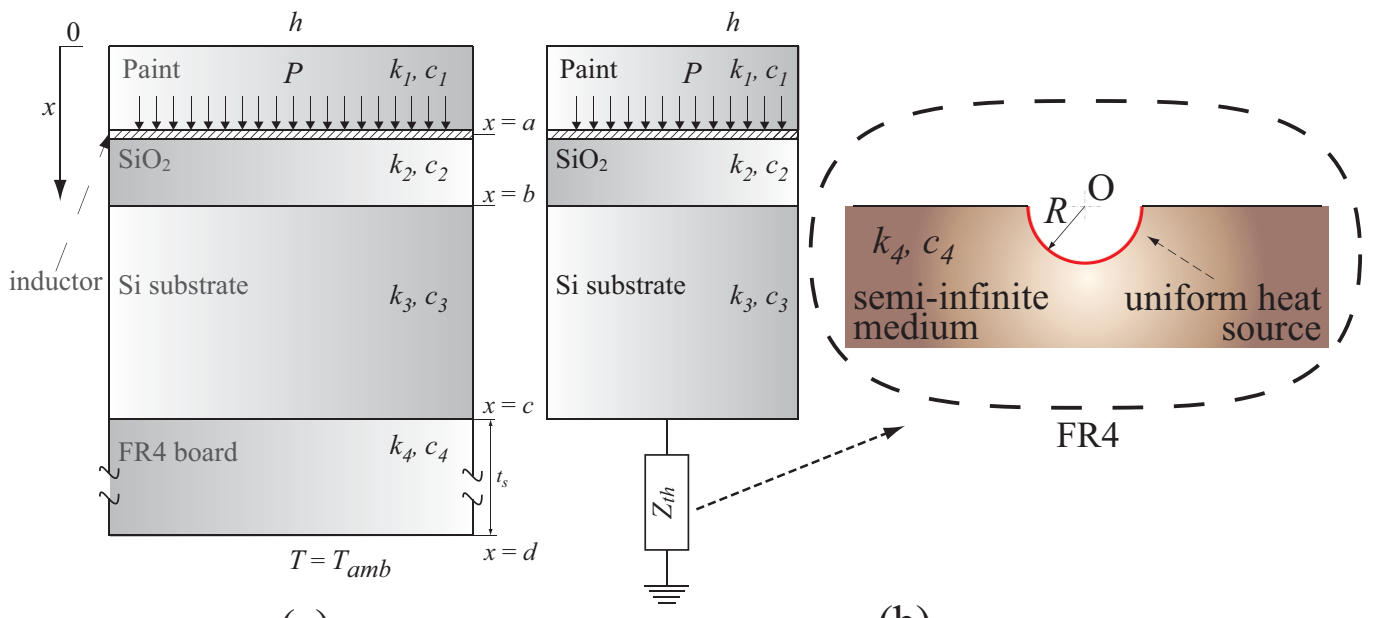

(a)

(b)

Fig. 2. (a) One-dimensional representation of the four-layered structure's thermal network, (b) Thermal impedance of the FR4 board used as boundary condition

The temperature response was then measured with the use infrared thermographic camera from CEDIP (Cedip Titanium 560M) [15]. The spectral response of this InSb camera is 3.6-4.9 $\mu \mathrm{m}$, and its noise equivalent temperature difference (NETD) is less than $25 \mathrm{mK}$ at $25^{\circ} \mathrm{C}$. The frame rate of the CEDIP camera was 700 frames per second. The option of sub-windowing was used for this purpose. In comparison with previous research [2], the frame rate is considered better, as it is considerably higher. Consequently, the results were transformed in the frequency domain and represented as Nyquist plots [3].

In order to guarantee a uniform surface and temporal emissivity, the surface of the sample has been painted with black mat paint. The exact thickness and other interface properties between the surface of the device and the paint mass cannot be evaluated directly. The paint layer has been assumed to be approximately $30 \mu \mathrm{m}$ thick. Hence during the experiments, the temperature on the top of the paint coating has been recorded. Therefore, this effect had to be taken into account throughout the analytical calculations and modelling. The value for the paint thickness used during the analysis is given in Table 1.

Specially designed extension rings have been used so as to achieve the required spatial resolution and render the small inductors visible on the infrared images. This way close up thermography was rendered possible [16, 17]. A 60 mm-extension tube was added between the lens and the camera housing, allowing the increase of the spatial resolution from about $88 \mu \mathrm{m}$ per pixel to $7 \mu \mathrm{m}$ per pixel. One image corresponds to $1.11 \mathrm{~mm} \times 0.9 \mathrm{~mm}$ on the silicon chip, while one pixel corresponds to $7 \times 7$ square micrometers on the silicon chip.

The results are presented in figure 3(a) in logarithmic scale. The temperature is averaged over the surface of the coil and then plotted versus time. Then the results were transformed in the frequency domain as described in section 2. The impedance function can be visualized in the complex plane using the Nyquist representation. The Nyquist plot is a curve where the $\operatorname{imag}\left(Z_{t h}(j \omega)\right)$ is plotted versus the real $\left(Z_{t h}(j \omega)\right)$ using angular frequency $\omega$ as a parameter.

The results obtained after transformation in the frequency domain are presented in figure 3(b). As explained by Kawka [3], a Nyquist plot can be analysed in a series of circles centred on the Ox axis. In special cases, the centre of these circles can lie above the $O x$ axis. The frequencies corresponding to the centres of the circles are in direct correlation to the time constant spectrum central characteristic values.

The thermal behaviour of all three inductors is similar. There are differences which are probably caused by the variations in the thickness of the layers as regards the heat flow path, or the positioning of the inductors on the die. On the other hand, as one would expect their response to a heat step function is similar. The frequencies where the central points of the main circular arcs are occurring are almost the same for all the three inductors.

Thanks to the high frequency rate of the thermal imaging equipment, it was rendered possible to observe the high frequency arc of the Nyquist plot. The high frequency arc of the diagram is mainly dependent on the material properties and geometry of the thermal network components in the vicinity of the heat sources. As regards the shape of the high frequency arc, it is obvious that the real part of the thermal impedance can take negative values. This is due to the paint coating which covers the heat source. Thus, the extracted thermal impedance can be described as transfer thermal impedance [18].

The low frequency arc covering the biggest part of the Nyquist plot is caused by the low thermal conductivity of the epoxy FR4 board. One can assume that the most defining factor of the thermal behaviour of the device is the convective cooling. The differences among the different curves are mainly concerning the size of the arcs, while their shape is almost the same. This can be owed to the positioning of the inductor with the highest thermal resistance closer to the centre of the silicon die, leading to a smaller convective surface in the vicinity of the heat source. On the contrary, the inductor with the lowest thermal resistance is inductor 1 which lies at the corner of the sample. This also advocates for validity of the measurements. 


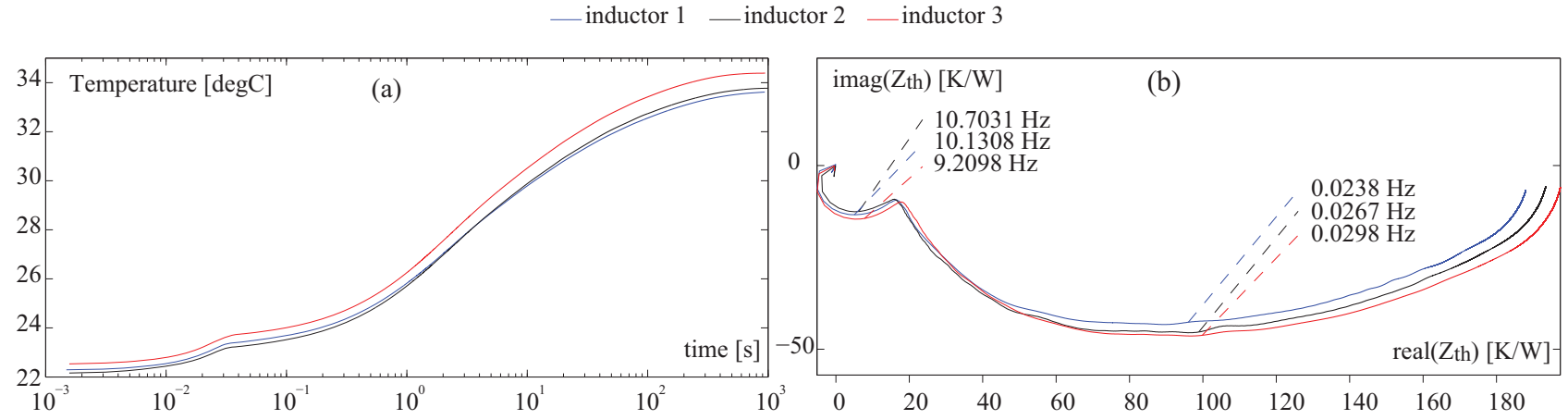

Fig. 3. (a) Temperature response for inductors 1, 2, and 3, (b) Nyquist plot of the thermal impedance.

\section{Analytical model}

A simple way to describe a device's dynamic thermal behaviour is with the use of one-dimensional heat equations. We consider the heat source's and each layer's surface equal (figure 2(a)). Therefore, the temperature is dependent on only one spatial coordinate $x$. The structure consists of four layers; the heat source is at the interface between the paint and silicon dioxide layer. In each layer, the temperature must satisfy the heat equation. The thickness of the paint layer is $a$, the one of the silicon dioxide is $b$-a, the thickness of the silicon layer is $c-b$, and that of the FR4 board is $t_{s}$. In the frequency domain, the system of equations that describes the heat diffusion throughout the device is:

$$
j \omega T_{i}(\omega, x)-k_{i} \frac{d^{2} T_{i}(\omega, x)}{d^{2} x}=0, x_{i}<x<x_{i}^{\prime},
$$

where the temperature $T_{i}$ can take the values $T_{1}, T_{2}, T_{3}$, and $T_{4}$ for layer $1,2,3$, and 4 respectively. The boundaries $\left(d_{i}, d_{i}^{\prime}\right)$ are $(0, a),(a, b),(b, c)$, and $(c, d)$ for the corresponding layers. $k_{i}$ is the thermal conductivity of the $i^{\text {th }}$ layer. Considering zero ambient temperature, the boundary condition on a surface where heat convection is present is given by:

$$
h T(L)-\left.k \frac{d T(x)}{d x}\right|_{x=L}=0
$$

where $L$ is the position of the boundary on the $x$ axis. In the current case the value of $L$ can be zero or $c+t_{s}$. Boundary conditions describing interfaces between layers are given by the equations:

$$
\begin{array}{r}
T_{i}\left(d_{i}\right)=T_{i+1}\left(d_{i}\right), \\
\left.k_{i} \frac{d T_{i}}{d x}\right|_{x=d_{i}}=\left.k_{i+1} \frac{d T_{i+1}}{d x}\right|_{x=d_{i}},
\end{array}
$$

where $d_{i}$ is the position of the interface between layer $i$ and $i+1$ on the $x$ axis. The boundary condition at the paint-silicon interface $\left(d_{i}=a\right)$, where the heat source is located, is described by the equation:

$$
\left.k_{1} \frac{d T_{1}}{d x}\right|_{x=a}-\left.k_{2} \frac{d T_{2}}{d x}\right|_{x=a}=P
$$

where $P$ is the power dissipated in the structure.

The results of the analysis are presented in form of Nyquist plots in figure 4 (model 1). One can notice that the graph consists of two arcs, one big in the low frequency range with central frequency at $\omega=0.0011 \mathrm{~Hz}$, and a smaller arc closer to centre of the axis. The value of the thermal resistance and the shape of the graph are not close to that of the experimental measurements of figure $3(\mathrm{~b})$.

If we assume that the epoxy FR4 layer is replaced by a thermal impedance boundary condition at $x=c$, then the differential equation (2) for the region $c<x<d$, and the boundary conditions (4) and (5) for $d_{i}=c$ can be replaced by a single boundary condition. In order to achieve that, we propose that the FR4 board is replaced by a semi-infinite medium made 
out of the same material. In addition, the interface boundary condition between the silicon layer and the FR4 board can be considered a semi-spherical surface with radius $R$, where the heat is dissipated uniformly from one medium to the other. A cross section of the proposed structure replacing the FR4 board is presented in figure 2(b). According to [4], the thermal impedance of such a structure is given by:

$$
Z_{t h}(j \omega)=\frac{Z_{0}}{1+\sqrt{j \omega / s_{0}}}=\frac{1}{2 \pi k_{4} R} \frac{1}{1+\sqrt{j \omega C_{4} / k_{4}}},
$$

where $Z_{0}=1 / 2 \pi k_{4} R$, and $s_{0}=k / C_{4} R^{2}$. The thermal impedance of the structure at the semi-spherical surface is given by:

$$
Z_{t h}(j \omega)=\frac{\left.T_{3}(j \omega)\right|_{x=c}}{P(j \omega)}
$$

After simple manipulations, the boundary condition at the boundary $x=c$ turns to:

$$
\left.T_{3}(j \omega)\right|_{x=c}=-\left.2 \pi k_{3} R^{2} \frac{d T_{3}(j \omega)}{d x}\right|_{x=c} \frac{1}{2 \pi k_{4} R\left(1+\sqrt{j \omega C_{4} / k_{4}}\right)} .
$$

Considering the work of Vermeersch [4], the thermal impedance proposed there fits well the thermal impedance proposed by equation (9). Without loss in validity, the boundary condition at $x=c$ can be described by:

$$
\left.T_{3}(j \omega)\right|_{x=c}=-\left.\pi k_{3} R^{2} \frac{d T_{3}(j \omega)}{d x}\right|_{x=c} \frac{1}{2 \pi k_{4} R\left(1+\sqrt{j \omega C_{4} / k_{4}}\right)} .
$$

After solving the new system of equations, we get the thermal impedance as plotted in figure 4 (model 2). The complex thermal impedance is calculated on top of the paint layer as mentioned earlier. Notice that the thermal resistance is reduced to almost half in comparison to the solution produced by the system of equations (2)-(6). On the contrary, the frequency where the minimum of the imaginary part occurs is multiplied. The shape of the arcs does not resemble a circular any more. It is shifted towards the imaginary axis. One should also notice that no other arc is any more visible.

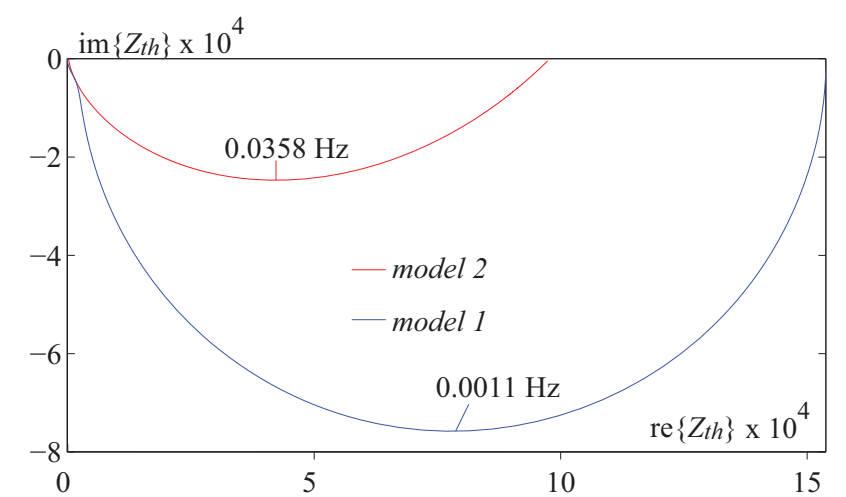

Fig. 4. Thermal impedance Nyquist plot of (model 1) the system of eq.(2)-(6), and (model2) the system where the differential equation (2) for $c<x<d$ and the boundary conditions at $x=c, d$ are replaced by equation (10) at $x=c$.

\section{Conclusion}

The main purpose of this work was to evaluate the dynamic thermal behaviour from thermographic measurements against analytical modelling results. The proposed models were based on simple one-dimensional set of diffusion equations representing the thermal network of the complex structure of the silicon die.

Infrared thermographic equipment was used to measure the temperature response to a unit step function. The frame rate and thus the sampling rate were improved in comparison to previous works. It was rendered possible to generate images at a rate of 700 frames per second. The temperature on the top of the paint coating has been recorded during the experiments. Special extension tube rings were used to increase the spatial resolution of the images. 
The temperature measurements were transformed in the frequency domain. The temperature response to a heating step function $T_{\text {step }}(t)$ has been used to evaluate the thermal impedance versus frequency. The thermal impedance is then presented in the form of Nyquist plot.

The thermal behaviour of all three inductors is similar. There are differences which are caused by the variations in the thickness of the layers as regards the heat flow path, and the positioning of the inductors on the die. The central characteristic frequencies of the main circular arcs are occurring are almost the same for all the three inductors.

A simple analytical model of the heat transfer throughout the device's thermal network is taken into account. Further simplifications are taken into account in order to approximate the experimental measurements. The system of equations is reduced as the differential equation (2) for $c<x<d$ and the corresponding boundary conditions are replaced by a simple differential equation (10), substituting the fourth layer by a surface impedance boundary condition. The correction introduced by this approach is improving the shape of the Nyquist plot in comparison to the experimental measurements.

The main discrepancy is the value of the total thermal resistance and the fact that the high frequency arc of the Nyquist plot in figure 4 (model 2) is not visible. Further modifications are needed for the analytical one-dimensional model to describe sufficiently the heat flow through the samples examined. Spreading in lateral directions is to be considered in future work.

\section{REFERENCES}

[1] AMIS, Ami semiconductor c5f/n - eeprom technology, technology specification sheet, Europractice IC Service, 2003.

[2] Kaluza M., Papagiannopoulos I., Wiecek B., Chatziathanasiou V., Hatzopoulos A., De Mey G., "Thermal characterization of silicon integrated spiral inductors by thermal impedance using transient thermography", Proceedings of 10th Quantitative InfraRed Thermography conference (X. Maldague, ed.), paper QIRT 2010064, Québec (Canada), 2010.

[3] Kawka P., De Mey G., Vermeersch B., "Thermal characterization of electronic packages using the nyquist plot of the thermal impedance", IEEE Transactions on Components and Packaging Technologies, vol. 30, pp.660665, 2007.

[4] Vermeersch B., "Thermal AC modelling, simulation and experimental analysis of microelectronic structures including nanoscale and high-speed effects", PhD thesis, Faculteit Ingenieurswetenschappen (TW) UGent, Gent, 2009.

[5] Vermeersch B., De Mey G., "Non-fourier thermal conduction in nano-scaled electronic structures", Proceedings of the International Conference Mixed Design of Integrated Circuits and System, 2006., pp. 431-436, 2006.

[6] Vermeersch B., De Mey G., "Dependency of thermal spreading resistance on convective heat transfer coefficient", Microelectronics Reliability, vol. 48, no. 5, pp. 734-738, 2008.

[7] Ellison G., "Maximum thermal spreading resistance for rectangular sources and plates with nonunity aspect ratios", IEEE Transactions on Components and Packaging Technologies, vol. 26, pp. 439 - 454, 2003.

[8] Rhee J., Bhatt A., "Spatial and temporal resolution of conjugate conduction-convection thermal resistance", IEEE Transactions on Components and Packaging Technologies, vol. 30, pp. 673 - 682, 2007.

[9] Luo X., Maoa Z., Liub S., "Analytical thermal resistances model for eccentric heat source on rectangular plate with convective cooling at upper and lower surfaces", International Journal of Thermal Sciences, vol. 50, p. 2198-2204, 2011.

[10] Szkely V., "Enhancing reliability with thermal transient testing", Microelectronics Reliability, vol. 42, p. 629-640, 2002.

[11] Janicki M., Banaszczyk J., Vermeersch B., De Mey G., Napieralski A., "Generation of reduced dynamic thermal models of electronic systems from time constant spectra of transient temperature responses", Microelectronics Reliability, vol. 51, p. 1351-1355, 2011.

[12] Papagiannopoulos I., Chatziathanasiou V., Hatzopoulos A., Kaluza M., Wiecek B., De Mey G., "Thermal analysis of integrated spiral inductors", Infrared Physics \& Technology, Volume 56, p. 80-84, 2013

[13] Vermeersch B., De Mey G., "Thermal impedance plots of micro-scaled devices", Microelectronics Reliability, vol. 46, pp. 174-177, 2006.

[14] Kydonopoulos A., "Simulation of the dynamic thermal behaviour of medium voltage cables with the use of the program pspice", Master's thesis, Aristotle University of Thessaloniki, Polytechnic School, Dept. of Electrical and Computer Engineering, 2010.

[15] Cedip Infrared Systems, DC019U-A TITANIUM User Manual.

[16] De Mey G., Wiecek B., Kaluza M., Olbrycht R., Strakowski R., Strakowska M.,"Importance of radiative heat transfer for infrared thermography measurements", Pomiary Automatyka Kontrola, vol. 57, pp. 1112-1115, 2011.

[17] Kaluza M., "Problem nagrzewania pierscieni posrednich stosowanych w badaniach termowizyjnych", Pomiary Automatyka Kontrola, vol. 57, pp. 1238-1241, 2011.

[18] Kawka P., De Mey G., Vermeersch B., "Thermal Characterization of Electronic Packages Using the Nyquist Plot of the Thermal Impedance", IEEE Transactions on Components and Packaging Technologies, vol. 30, p. 660665, 2007 